\title{
Reaction of Sulfur and Sustainable Algae Oil for Polymer Synthesis and Enrichment of Saturated Triglycerides
}

\author{
Adarsha Gupta, ${ }^{\text {a, }}$ Max J. H. Worthington,,${ }^{b}+$ Munish Puri, ${ }^{a,}{ }^{,}$and Justin M. Chalkerb,
}

a) Medical Biotechnology, Centre for Marine Bioproducts Development, College of Medicine and Public Health, and Flinders Health and Medical Research Institute, Flinders University, Bedford Park, South Australia, 5042, Australia

b) Institute for Nanoscale Science and Technology, College of Science and Engineering, Flinders University, Bedford Park, South Australia, 5042, Australia

*To whom correspondence should be addressed:

munish.puri@flinders.edu.au

justin.chalker@flinders.edu.au

t These authors contributed equally to this publication

\begin{abstract}
There is growing interest in the bio-based production of lipids from algae. These lipids have a range of uses including nutritional supplements and precursors to biodiesel. Single-cell thraustochytrids are especially attractive in this regard in that they can produce over $50 \%$ of their weight as triglycerides. Furthermore, the distribution of saturated and unsaturated triglycerides can be modulated by changes in strain variation and modulation of fermentation conditions. Nonetheless, there remains a need for versatile downstream processing to enrich these so-called "single cell oils" into classes based on degree of unsaturation. In this study, we report a novel strategy for enriching saturated triglycerides produced in thraustochytrids. The method features direct reaction of elemental sulfur with the algae oil extract. The sulfur copolymerizes with $>90 \%$ of the unsaturated triglycerides, providing a new route to a class of materials previously used in environmental remediation, Li-S battery cathodes, slow-release fertilisers, and insulation. The unreacted oil is enriched in saturated triglycerides, which can be isolated by extraction for potential use in biodiesel production. In this way, a single batch of sustainably produced algae oil can be converted into multiple useful products in a single step.
\end{abstract}

\section{INTRODUCTION}

There has been growing interest in microalgal cultivation to produce high value compounds with potential applications in human health, livestock, aquaculture and biofuels. ${ }^{1}$ Compounds derived from triglycerides and lipids in microalgae are of particular interest as these substances can comprise 20$70 \%$ of the dry mass of the organism. ${ }^{1}$ However, due to high costs involved in lipid downstream processing, biorefinery platforms typically produce other important co-products such as polysaccharides, proteins, terpenes, phenolics and alkaloids to offset the oil production costs. ${ }^{1}$ For lipid-intensive production, single cell oils from cultured microalgae are of particular interest as they offer an alternative to plant-based lipids that require land use. ${ }^{1}$ In this context, heterotrophic thraustochytrids could be a potential source of useful tryglycerides and due to their amenability to the tuning of the oil profile through various fermentation and metabolic influences. In this way, these organisms can be engineered to produce the fatty oil profile of plant oils. Furthermore, thraustochytrids can produce high value nutritional oil containing omega-3 fatty acids, such as docosahexaenoic acid (DHA) mainly in the form of triglycerides. ${ }^{2}$ Remarkably, triglycerides accumulated in thraustochytrids constitute more than $90 \%$ of the lipids, ${ }^{3}$ which can be used as high value nutritional supplements for humans. In addition, low cholesterol content makes thraustochytrid oil desirable for supplementing food products. ${ }^{4}$ Thraustochytrid derived-oil has been designated 'generally recognized as safe' status for its use in food products. ${ }^{5}$ In other applications, thraustochytrid oil containing triglycerides has been produced as a precursor for biodiesel production. 
The ability to produce high amounts of saturated fatty acids and monounsaturated fatty acids is particularly important in this context. ${ }^{6}$ Despite these preliminary successes and promising outlook for lipids produced from thraustochytrid algae, it is still necessary to develop flexible methods for downstream processing to enrich oils with a desired degree of saturation or unsaturation. It is also of interest to develop new products from these oils given the growing interest in their sustainable production. $^{7}$

In this report, we address both of these issues through a sulfur-based reactive-processing of triglycerides produced in thraustochytrid microalgae. Specifically, sulfur was reacted with the mixture of the algae-derived triglycerides, forming a novel copolymer with the unsaturated triglycerides. The resulting polymer was analogous to related materials made from unsaturated plant oils, which have been used in a wide range of applications including heavy metal remediation, ${ }^{8-11}$ oil spill clean-up, ${ }^{12}$ PFAS capture, ${ }^{13}$ slow-release fertilisers, ${ }^{14,15}$ composite synthesis, ${ }^{16}$ cathode materials for Li-S batteries, ${ }^{17}$ and thermal insulation. ${ }^{18}$ Accessing these materials through a new triglyceride source is therefore important, as it does not require access to plant oils otherwise used for food or those that require significant and detrimental land use. Additionally, the remaining saturated triglycerides that did not react with sulfur could be recovered in a significantly enriched form. This chemistry therefore also constitutes a novel method for separating saturated and unsaturated triglycerides produced in algae - an important capability for the downstream use of these triglycerides in food products and biodiesel production. Additionally, the process generates valuable sulfur polymers in the same step. Importantly, the low-cost and high abundance of sulfur bodes well for this method in terms of scalability and sustainability. The overall strategy is summarized in Figure 1.

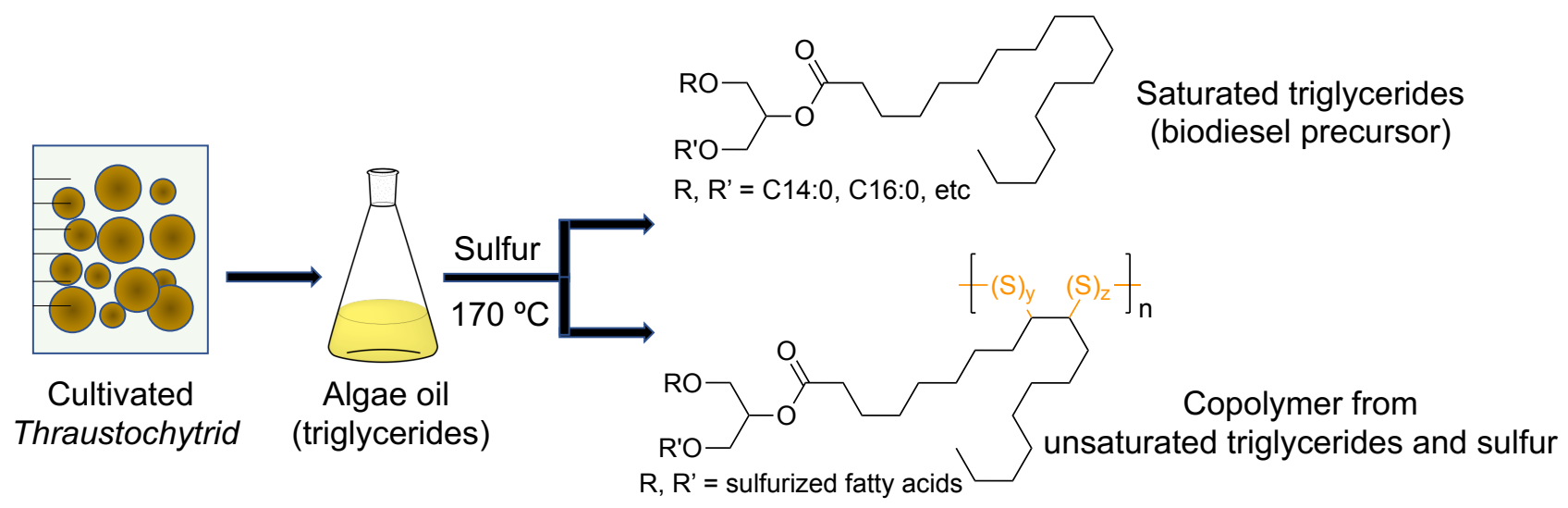

(cathode material, metal sorbent, insulation, fertilizer)

Figure 1. Reacting thraustochytrid-derived triglycerides with sulfur provides novel polymer materials and enriched saturated triglycerides. The strategy features sustainable, cultured triglyceride biosynthesis, and a novel divergent processing method to provide saturated triglycerides and functional sulfur polymers. The saturated triglycerides are established precursors to biodiesel. ${ }^{6}$ Copolymers made from the reaction of sulfur and unsaturated triglycerides have a number of established uses in energy storage, metal binding, insulation, fertilizers, and more. ${ }^{19}$

\section{MATERIALS AND METHODS}

Full experimental details and characterization are provided in the online Supporting Information.

\section{Biomass Production}

An in-house algae strain (Schizochytrium sp. MASA\#4) was grown in a 6 L bioreactor (Infors HT, Invitrogen Technologies) to produce the biomass required for this study. The inoculum was prepared in medium containing glycerol $(10 \mathrm{~g} / \mathrm{L})$, yeast extract $(1 \mathrm{~g} / \mathrm{L})$, peptone $(1 \mathrm{~g} / \mathrm{L})$ and incubated for $48 \mathrm{~h}$ at $25{ }^{\circ} \mathrm{C}$ with agitation at $150 \mathrm{rpm}$. Inoculum $(10 \%)$ was used in the production medium containing glycerol $120(\mathrm{~g} / \mathrm{L})$, yeast extract $(10 \mathrm{~g} / \mathrm{L})$, peptone $(1 \mathrm{~g} / \mathrm{L}), \mathrm{MgSO}_{4}(10 \mathrm{~g} / \mathrm{L})$, and sodium acetate $(4$ $\mathrm{g} / \mathrm{L}$ ) in a bioreactor and cultivated at $25{ }^{\circ} \mathrm{C}$ for 5 days. The $\mathrm{pH}$ of the medium was maintained at 6.5 using potassium hydroxide solution. The culture medium was harvested after 5 days and subjected to freeze drying to obtain dried biomass. 


\section{Lipid extraction}

Freeze dried biomass $(2.0 \mathrm{~g})$ was used for lipid extraction using homogenization in a chloroform: methanol mixture $(2: 1, \mathrm{v} / \mathrm{v})$. The homogenization was repeated three times and the extracts were combined and filtered through a $0.22 \mu \mathrm{m}$ filter. The solvent was then removed using a rotary evaporator (bath temperature $=50^{\circ} \mathrm{C}$ ) and lipid weight was determined gravimetrically. ${ }^{20}$

\section{Conversion of triglycerides to fatty acid methyl esters (FAMEs) and GC analysis}

Lipids were transesterified and fatty acid methyl esters (FAMEs) were analysed using a previously described method. ${ }^{20}$ FAMEs analysis was performed on a Shimadzu Gas chromatography (GC, 2090N) equipped with flame ionisation detector (FID) and connected to a BID 2030 unit using FAMEWAX column (30 m x $0.32 \mathrm{~mm}$ ID (inner diameter)). The inlet was held at $25^{\circ} \mathrm{C}$ with a constant column flow rate at $5 \mathrm{~mL} / \mathrm{min}$ with split injection $(1 / 150)$. The oven program was held at $150{ }^{\circ} \mathrm{C}\left(5 \mathrm{~min}\right.$. hold), ramped to $250^{\circ} \mathrm{C}$ at a rate of $10^{\circ} \mathrm{C}$ per min. and held at $250{ }^{\circ} \mathrm{C}$ for $1 \mathrm{~min}$. Fatty acid esters were quantified by comparison of peak areas of authentic FAMEs standards (Sigma Aldrich CRM47885).

\section{Hydrogenation of algae oil}

Algae oil (409 mg) was dissolved in $15 \mathrm{~mL}$ ethanol in a $25 \mathrm{~mL}$ round bottom flask. Palladium on carbon $(20 \mathrm{mg}, 10 \mathrm{wt} . \% \mathrm{Pd})$ was added, the flask sealed with a septum and sparged with hydrogen gas for 20 minutes. The mixture was left to react at room temperature with magnetic stirring for 3 days under an atmosphere of hydrogen ( $1 \mathrm{~atm}$, balloon). The mixture was then filtered over celite $\left(\mathrm{SiO}_{2}\right)$ under vacuum to remove the catalyst and dried to isolate the oil. The average yield of isolated hydrogenated oil from three trials was $261 \mathrm{mg}$ (72\% yield).

\section{Copolymerization of algae oil with sulfur}

Algae oil (409 mg) was heated to $170{ }^{\circ} \mathrm{C}$ with stirring in a $25 \mathrm{~mL}$ round bottom flask. After 5 minutes, the algae oil turned from a viscous orange liquid to dark brown and free flowing liquid. Elemental sulfur $(409 \mathrm{mg})$ was added slowly over 5 minutes to keep the mixture at approximately $170{ }^{\circ} \mathrm{C}$. A heat gun was used to melt sulfur adhering to the upper walls of the flask. After 30 minutes the viscosity increased and stirring was slowed to ensure continual mixing. Approximately 5 minutes after the increase in viscosity, the reaction mixture could not be stirred. After a further 10 minutes at $170{ }^{\circ} \mathrm{C}$, the flask was removed from the oil bath and allowed to cool to room temperature. After 1 hour of cooling the material had vitrified into a dark brown rubber. The synthesis was carried out in triplicate. The average amount of product formed in these reactions was $458 \mathrm{mg}$ of solid product and $146 \mathrm{mg}$ of unreacted oil (obtained by extraction into ethanol). Some unreacted sulfur was embedded in the polymer (see below) and some was lost to sublimation during the reaction. The isolated copolymer was then characterized by scanning electron microscopy (SEM), simultaneous thermal analysis, dynamic scanning calorimetry, ${ }^{1} \mathrm{H}$ NMR spectroscopy and powder X-ray diffraction, as detailed in the Supporting Information.

\section{Analysis of algae oil recovered after hydrogenation or after copolymerization with sulfur \\ After the hydrogenation process, $10 \mathrm{mg}$ of the oil sample was directly used for transesterification and GC analysis following the FAME analysis cited above. However, after the sulphur polymerization process, the polymer containing oil was subjected to ethanol extraction $(20 \mathrm{~mL})$ at $50^{\circ} \mathrm{C}$ for 2 hours. The ethanol in the solvent extract was removed by rotary evaporation at $50{ }^{\circ} \mathrm{C}$, and lipid weight was determined gravimetrically. $10 \mathrm{mg}$ of this dried lipid was used for transesterification and GC analysis.}

\section{RESULTS AND DISCUSSION}

Thraustochytrid oil can be used a feedstock for various industrial applications, such as biodiesel production, nutrition supplements, and aquaculture feed. The heterotrophic thraustochytrids offer various advantages when compared to plants and photoautotrophic algae sources, which include 
controlled fermentation, utilization of different nutrient sources, and limited land use. The result is a more sustainable option for triglyceride production with high biomass and lipid accumulation. Thraustochytrids can grow on a variety of carbon sources, including glucose, glycerol, other sugars, agricultural residues, and waste products, ${ }^{7}$ which is a further feature with regards to sustainability. In this study, glycerol was used a carbon source as it helps in the accumulation of more lipids in the cell bodies of thraustochytrids. ${ }^{21}$ High biomass $(28 \mathrm{~g} / \mathrm{L})$ was obtained upon using glycerol as carbon source. The lipid content in the biomass was found to be $50 \%$ of the dry weight, which is consistent with previous studies. ${ }^{2}$ The main objective of this study was to produce single cell oil rich in triglycerides and polyunsaturated fatty acids and then develop a novel downstream processing method involving the direct reaction of the algae oils with sulfur. The aim was to have the sulfur react with the unsaturated triglycerides, $, 8,9,17$ forming a polymer in a process analogous to the inverse vulcanization reaction. ${ }^{19,22-25}$ This route to sulfur-triglyceride copolymers would be complementary to other approaches such as the copolymerization of sulfur and algae-derived monomers such as triterpene botryococcene. ${ }^{26}$ Furthermore, many applications of these polymers in environmental remediation, ${ }^{8-13}$ energy storage, ${ }^{17}$ slow-release fertilizers, ${ }^{14,}{ }^{15}$ composite synthesis,${ }^{16}$ and insulation ${ }^{18}$ are already established, so establishing another sustainable route to these macromolecules is important. Finally, it was anticipated that any saturated algae oil could be recovered in an enriched form after the reaction with sulfur. Such triglycerides with high levels of saturation are common precursors to biodiesel.

The thraustochytrid strain was first cultured in a 6 L bioreactor with glycerol as the primary carbon source. After cultivation for 5 days, the biomass was harvested and lyophilized to obtain dry biomass. The oils from a portion of this biomass $(2.0 \mathrm{~g})$ were then extracted by homogenization in a mixture of chloroform and methanol (2:1 by volume), based on a previously published protocol. ${ }^{20}$ Three extractions were combined, filtered, and concentrated under reduced pressure to provide approximately $1.0 \mathrm{~g}$ of oil (50\% of the dry biomass). This oil was then subjected to a transesterification process to convert the triglycerides to their corresponding fatty acid methyl esters (FAMEs). The FAMEs were then analyzed by gas chromatography and identified by comparison to known standards. The most prominent fatty acids detected were the methyl esters of palmitic acid (C16:0) at 41\% abundance relative to the total fatty acid content, docosahexaenoic acid (DHA, C22:6n3) at 29.0\%, and docosapentaenoic acid (DPA, 22:5n6) at 11.7\%. The relative abundance of saturated fatty acids, monounsaturated fatty acids and polyunsaturated fatty acids (PUFAs) in the microalgal oil triglycerides were $54 \%, 1 \%$, and $45 \%$, respectively (Figure 2 ).

Thraustochytrids are known for the accumulation of long chain PUFAs, and in particular the omega-3 fatty acids such as DHA, but the accumulation of a relatively high level saturated fatty acids in this culture indicated that this oil could be potentially useful as a precursor to biodiesel. This is an important finding as the use of saturated fatty acid triglycerides from microbial oil is relatively underexplored. We therefore turned to methods to enrich the unsaturated fraction of the microalgal oil. The first strategy was as classic catalytic hydrogenation. Accordingly, the algae oil was dissolved in ethanol before adding a palladium catalyst $(20 \mathrm{mg} \mathrm{Pd} / \mathrm{C}$ for $409 \mathrm{mg}$ of algae oil). The reaction was then sparged with hydrogen and reacted further under one atmosphere of hydrogen for 3 days. The experiment was repeated in triplicate with an average isolated yield of $72 \%$ after recovering the product by filtrations through celite. FAMEs analysis was carried out on the product, revealing complete consumption of polyunsaturated components of the oil, but with $21 \%$ of the analyzed methyl esters presenting as monounsaturated species (Figure S1 and S2). This result was consistent with ${ }^{1} \mathrm{H}$ NMR analysis of the algae oil and products of hydrogenation (Figures S3-S5). This hydrogenation method provides a straightforward way for converting the algae oil mixture to mono- and unsaturated triglycerides, but the reaction was not complete even after extended reaction time. More importantly, this experiment provides a benchmark for comparison to the proposed sulfur method, which was investigated next.

Accordingly, the reaction of the algae oil with sulfur was evaluated for production of novel sulfur polymers (through reaction with unsaturated triglycerides) with concomitant enrichment of unreacted saturated triglycerides. Accordingly, the algae oil $(409 \mathrm{mg})$ was first pre-heated to $170{ }^{\circ} \mathrm{C}$ 
before an equal mass of elemental sulfur was added portionwise over 5 minutes. The reaction was stirred for 30-35 minutes, after which time the reaction mixture became increasingly viscous. After an additional 10 minutes of reaction time, the mixture was cooled to room temperature to provide a brown solid. Unreacted oil was extracted by stirring with ethanol at $50{ }^{\circ} \mathrm{C}$ for 2 hours. The solid material was then isolated by filtration ( $458 \mathrm{mg}$ recovered) and the filtrate was concentrated under reduced pressure to provide the unreacted oil (146 mg).

The recovered oil was then converted to the corresponding fatty acid methyl esters via transesterification for subsequent $\mathrm{GC}$ analysis. The results (Figure 2) indicated that no polyunsaturated fatty acids remained in this mixture. This implies that sulfur reacted with the PUFAs and converted them into the insoluble polymer material. Furthermore, the saturated fatty acids were more effectively enriched than the benchmark hydrogenation method: $90 \%$ of this mixture was derived from saturated fatty acids and $<10 \%$ was derived from monounsaturated fatty acids (Figure 2 and Figures S6-S7). These results were corroborated by ${ }^{1} \mathrm{H}$ NMR analysis of the recovered algae oil (Figure S8). This means that the polyunsaturated triglycerides in the algae oil could be quantitatively converted into a copolymer through reaction with sulfur. The unreacted oil is comprised solely of saturated and monounsaturated species, both of which are suitable as biodiesel precursors. In this way, a single reaction of the crude algae oil with sulfur provides a novel polymer that is easily separable from a biodiesel precursor.

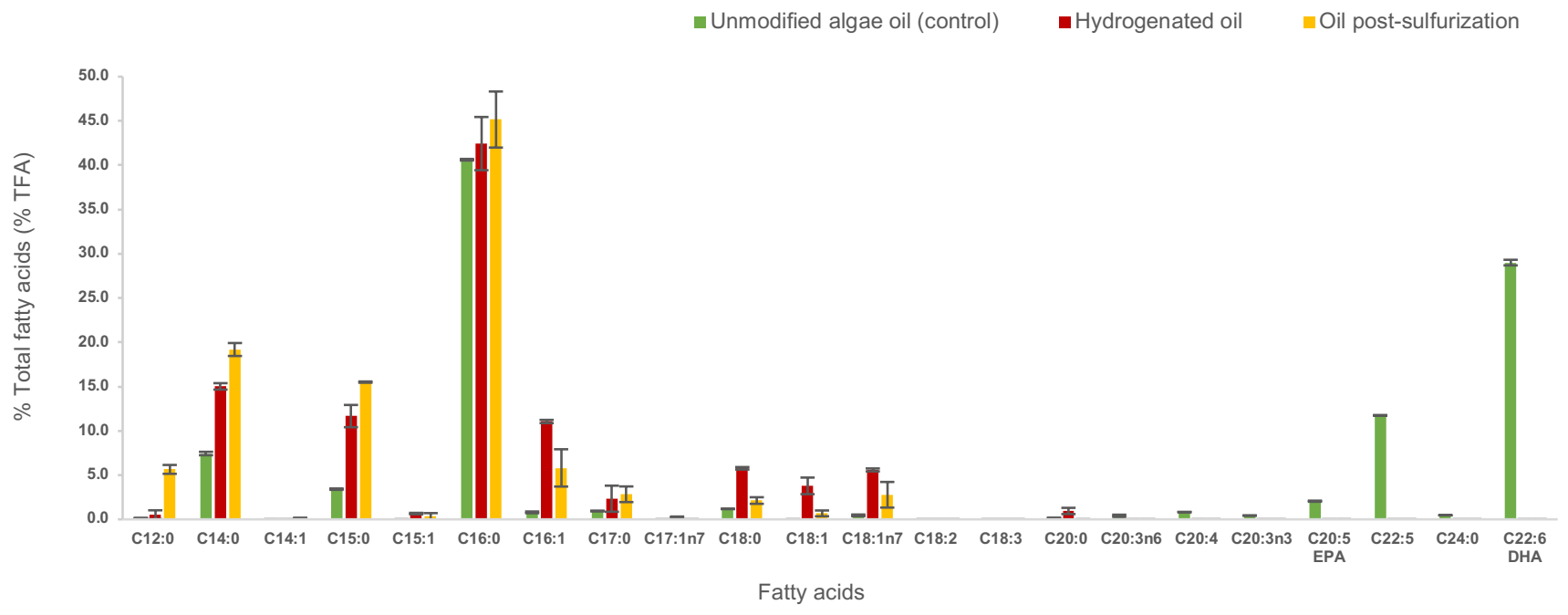

Figure 2. FAMEs analysis after transesterification of the original algae oil sample (control, green), the oil recovered after hydrogenation (red), and oil recovered after reaction of the algae oil with sulfur (orange). No polyunsaturated fatty acids (PUFAs) were detected after hydrogenation or after the reaction of the algae oil with sulfur.

The isolated polymer (Figure 3) was characterized next in order to compare its composition and properties to related polymers made from the reaction of sulfur with unsaturated vegetable oils. ${ }^{8}$, 9, 17 The material was qualitatively more brittle than these previously reported polymers. Scanning electron microscopy, energy dispersive X-ray analysis, simultaneous thermal analysis, and powder X-ray diffraction all revealed substantial amounts of crystalline sulfur (Figure S10-S12 and Figure S15). Differential scanning calorimetry was used to quantify this unreacted sulfur, indicating that the algae oil sulfur copolymer contains approximately $50 \mathrm{wt} \% \mathrm{~S}_{8}$ by mass. Therefore, it is appropriate to characterize this species as a composite material, similar to that observed in the reaction of large amounts of sulfur with unsaturated vegetable oils. ${ }^{8,17}$ These composites have found diverse applications such as use as cathode materials, ${ }^{17}$ mercury sorbents, ${ }^{8,9}$ insulating materials, ${ }^{18}$ and slow release fertilsers ${ }^{14,15}$ in which both the polymer and the $\mathrm{S}_{8}$ play a role in redox chemistry, mercury 
binding, thermal insulation, and nutrient release, respectively. In this study, a new route to this class of materials was established through sustainably produced algae oil.

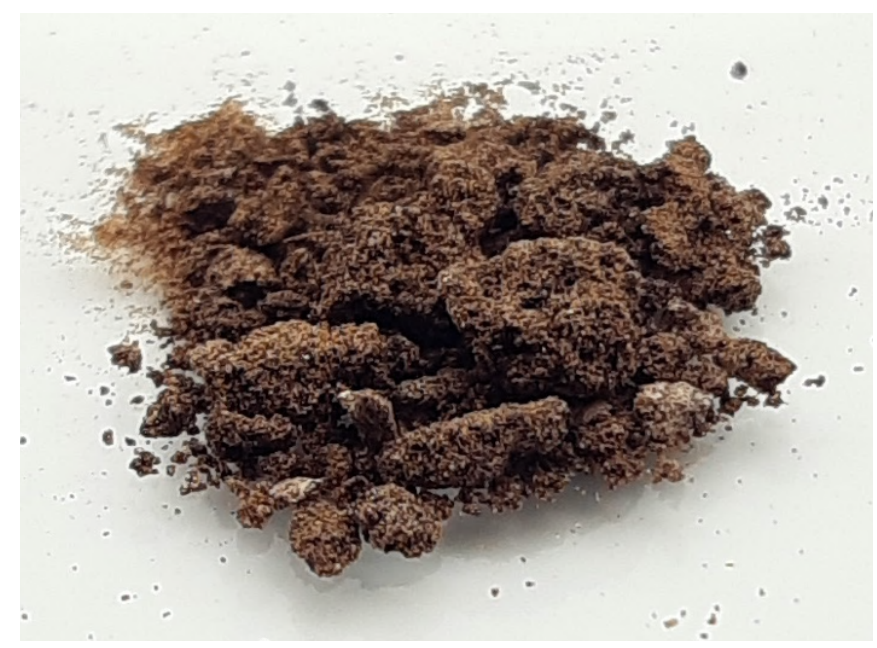

Figure 3. Polymer composite formed from the reaction of elemental sulfur with the polyunsaturated triglycerides in the thraustochytrid oil. This material contains both polymer and free elemental sulfur ( $\sim 50 \%$ based on calorimetric analysis). Related polymer composites with free sulfur have found use as cathode materials, ${ }^{17}$ mercury sorbents, ${ }^{8,9}$ insulating materials, ${ }^{18}$ and slow release fertilsers. ${ }^{14,} 15$ This is the first route to these materials from cultured algae oil.

\section{CONCLUSIONS}

Thraustochytrids are a valuable source of diverse triglycerides, composed of both saturated and unsaturated fatty acid components. This study demonstrated the production of such oils and a novel method to separate polyunsaturated triglycerides from monosaturated and unsaturated triglycerides. This process converted the polyunsaturated triglycerides into novel, sulfur-rich polymer composites. The unreacted saturated components were easily recovered through extraction with ethanol, for potential use as biodiesel precursors. Future studies will focus on upscaling the algae oil production and novel uses of the sulfur polymers reported in this study. With increasing interest in the synthesis and applications of sulfur-rich polymers, ${ }^{19}$ this study is an important step forward in the sustainable production of the triglyceride comonomer.

\section{ACKNOWLEDGEMENTS}

This research was supported by the Australian Research Council (DP200100090). The authors also thank Flinders University Industry Partnership Seed Grants (IPSG) 2020 for supporting collaborative research activity.

\section{REFERENCES}

1. Puri, M.; Gupta, A.; McKinnon, R. A.; Abraham, R. E., Marine bioactives: from energy to nutrition. Trends Biotechnol. 2021, In press. DOI: 10.1016/j.tibtech.2021.08.004.

2. Liu, Y.; Tang, J.; Li, J.; Daroch, M.; Cheng, J. J., Efficient production of triacylglycerols rich in docosahexaenoic acid (DHA) by osmo-heterotrophic marine protists. Appl. Microbiol. Biotechnol. 2014, 98, 9643-9652.

3. Lee-Chang, K. J.; Taylor, M. C.; Drummond, G.; Mulder, R. J.; Mansour, M. P.; Brock, M.; Nichols, P. D., Docosahexaenoic Acid Is Naturally Concentrated at the sn-2 Position in Triacylglycerols of the Australian Thraustochytrid Aurantiochytrium sp. Strain TC 20. Mar. Drugs 2021, 19, 382.

4. Burja, A. M.; Radianingtyas, H.; Windust, A.; Barrow, C. J., Isolation and characterization of polyunsaturated fatty acid producing Thraustochytrium species: screening of strains and optimization of omega-3 production. Appl. Microbiol. Biotechnol. 2006, 72, 1161-1169. 
5. Fossier Marchana, L.; Lee Chang, K. J.; Nichols, P. D.; Mitchellc, W. J.; Polglased, J. L.; Gutierrez, T., Taxonomy, ecology and biotechnological applications of thraustochytrids: A review. Biotechnol. Adv. 2018, 36, 26-46.

6. Chen, C.-Y.; Lee, M.-H.; Leong, Y. K.; Chang, J.-S.; Lee, D.-J., Biodiesel production from heterotrophic oleaginous microalga $\mathrm{T}$ Thraustochytrium sp. BM2 with enhanced lipid accumulation using crude glycerol as alternative carbon source. Bioresour. Technol. 2020, 306, 123113.

7. Gupta, A.; Barrow, C. J.; puri, M., Multiproduct biorefinery from marine thraustochytrids towards a circular bioeconomy. Trends Biotechnol. 2021, In press. DOI: 10.1016/j.tibtech.2021.09.003.

8. Worthington, M. J. H.; Kucera, R. L.; Albuquerque, I. S.; Gibson, C. T.; Sibley, A.; Slattery, A. D.; Campbell, J. A.; Alboaiji, S. F. K.; Muller, K. A.; Young, J.; Adamson, N.; Gascooke, J. R.; Jampaiah, D.; Sabri, Y. M.; Bhargava, S. K.; Ippolito, S. J.; Lewis, D. A.; Quinton, J. S.; Ellis, A. V.; Johs, A.; Bernardes, G. J. L.; Chalker, J. M., Laying Waste to Mercury: Inexpensive Sorbents Made from Sulfur and Recycled Cooking Oils. Chem. Eur. J. 2017, 23, 16219-16230.

9. Tikoalu, A. D.; Lundquist, N. A.; Chalker, J. M., Mercury sorbents made by inverse vulcanization of sustainable triglycerides: the plant oil structure influences the rate of mercury removal from water. Adv. Sustainable Syst. 2020, 4, 1900111.

10. Lundquist, N. A.; Worthington, M. J. H.; Adamson, N.; Gibson, C. T.; Johnston, M. R.; Ellis, A. V.; Chalker, J. M., Polysulfides made from re-purposed waste are sustainable materials for removing iron from water. $R S C A d v$. 2018, 8, 1232-1236.

11. Lundquist, N. A.; Chalker, J. M., Confining a spent lead sorbent in a polymer made by inverse vulcanization prevents leaching. Sustain. Mater. Techno. 2020, 26, e00222.

12. Worthington, M. J. H.; Shearer, C. J.; Esdaile, L. J.; Campbell, J. A.; Gibson, C. T.; Legg, S. K.; Yin, Y.; Nicholas A. Lundquist; Gascooke, J. R.; Albuquerque, I. S.; Shapter, J. G.; Andersson, G. G.; Lewis, D. A.; Bernardes, G. J. L.; Chalker, J. M., Sustainable Polysulfides for Oil Spill Remediation: Repurposing Industrial Waste for Environmental Benefit. Adv. Sustainable Syst. 2018, 2, 1800024.

13. Lundquist, N. A.; Sweetman, M. J.; Scroggie, K. R.; Worthington, M. J. H.; Esdaile, L. J.; Alboaiji, S. F. K.; Plush, S. E.; Hayball, J. D.; Chalker, J. M., Polymer Supported Carbon for Safe and Effective Remediation of PFOA- and PFOS-Contaminated Water. ACS Sustainable Chem. Eng. 2019, 7, 11044-11049.

14. Mann, M.; Kruger, J. E.; Andari, F.; McErlean, J.; Gascooke, J. R.; Smith, J. A.; Worthington, M. J. H.; McKinley, C. C. C.; Campbell, J. A.; Lewis, D. A.; Hasell, T.; Perkins, M. V.; Chalker, J. M., Sulfur polymer composites as controlled-release fertilizers. Org. Biomol. Chem. 2019, 17, 1929-1936.

15. Fortuna do Valle, S.; Soares Giroto, A.; Gestal Reis, H. P.; Guimarães, G. G. F.; Ribeiro, C., Synergy of Phosphate-Controlled Release and Sulfur Oxidation in Novel Polysulfide Composites for Sustainable Fertilization. J. Agric. Food Chem. 2021, 69, 2392-2402.

16. Lundquist, N. A.; Tikoalu, A. D.; Worthington, M. J. H.; Shapter, R.; Tonkin, S. J.; Stojcevski, F.; Mann, M.; Gibson, C. T.; Gascooke, J. R.; Karton, A.; Henderson, L. C.; Esdaile, L. J.; Chalker, J. M., Reactive compression molding post-inverse vulcanization: A method to assemble, recycle, and repurpose sulfur polymers and composites. Chem. Eur. J. 2020, 26, 10035-10044.

17. Hoefling, A.; Lee, Y. J.; Theato, P., Sulfur-Based Polymer Composites from Vegetable Oils and Elemental Sulfur: A Sustainable Active Material for Li-S Batteries. Macromol. Chem. Phys. 2017, 218, 1600303.

18. Bu Najmah, I.; Lundquist, N. A.; Stanfield, M. K.; Stojcevski, F.; Campbell, J. A.; Esdaile, L. J.; Gibson, C. T.; Lewis, D. A.; Henderson, L. C.; Hasell, T.; Chalker, J. M., Insulating composites made from sulfur, canola oil, and wool. ChemSusChem 2021, 14, 2352-2359. 
19. Chalker, J. M.; Worthington, M. J. H.; Lundquist, N. A.; Esdaile, L. J., Synthesis and Applications of Polymers Made by Inverse Vulcanization. Top. Curr. Chem. 2019, 377, 16.

20. Gupta, A.; Singh, D.; Byreddy, A. R.; Thyagarajan, T.; Sonkar, S. P.; Mathur, A. S.; Tuli, D. K.; Barrow, C. J.; Puri, M., Exploring omega-3 fatty acids, enzymes and biodiesel producing thraustochytrids from Australian and Indian marine biodiversity. Biotechnol. $J$. 2016, 11, 345-355.

21. Gupta, A.; Singh, D.; Barrow, C. J.; Puri, M., Exploring potential use of Australian thraustochytrids for the bioconversion of glycerol to omega-3 and carotenoids production. Biochem. Eng. J. 2013, 78, 11-17.

22. Chung, W. J.; Griebel, J. J.; Kim, E. T.; Yoon, H.; Simmonds, A. G.; Ji, H. J.; Dirlam, P. T.; Glass, R. S.; Wie, J. J.; Nguyen, N. A.; Guralnick, B. W.; Park, J.; Somogyi, A.; Theato, P.; Mackay, M. E.; Sung, Y.-E.; Char, K.; Pyun, J., The use of elemental sulfur as an alternative feedstock for polymeric materials. Nat. Chem. 2013, 5, 518-524.

23. Griebel, J. J.; Glass, R. S.; Char, K.; Pyun, J., Polymerizations with elemental sulfur: A novel route to high sulfur content polymers for sustainability, energy and defense. Prog. Polym. Sci. 2016, 58, 90-125.

24. Zhang, Y.; Glass, R. S.; Char, K.; Pyun, J., Recent advances in the polymerization of elemental sulphur, inverse vulcanization and methods to obtain functional Chalcogenide Hybrid Inorganic/Organic Polymers (CHIPs). Polym. Chem. 2019, 10, 4078-4105.

25. Worthington, M. J. H.; Kucera, R. L.; Chalker, J. M., Green chemistry and polymers made from sulfur. Green Chem. 2017, 19, 2748-2761.

26. Oishi, S.; Oi, K.; Kuwabara, J.; Omoda, R.; Aihara, Y.; Fukuda, T.; Takahashi, T.; Choi, J.-C.; Watanabe, M.; Kanbara, T., Synthesis and Characterization of Sulfur-Based Polymers from Elemental Sulfur and Algae Oil. ACS Appl. Polym. Mater. 2019, 1, 1195 1202. 